\title{
Lowering Tomography Costs in Quantum Simulation with a Symmetry Projected Operator Basis
}

\author{
Scott E. Smart and David A. Mazziotti* \\ Department of Chemistry and The James Franck Institute, \\ The University of Chicago, Chicago, IL 60637
}

(Dated: Submitted 7 August 2020, Published January 28, 2021. Phys. Rev. A 103, 012420.)

\begin{abstract}
Measurement in quantum simulations provides a means for extracting meaningful information from a complex quantum state, and for quantum computing, reducing the complexity of measurement will be vital for near-term applications. For most quantum simulations, the targeted state will obey several symmetries inherent to the system Hamiltonian. We obtain an alternative symmetry projected basis of measurement that reduces the number of measurements needed by a constant factor. Our scheme can be implemented at no additional cost on a quantum computer, can be implemented under various measurement or tomography schemes, and is reasonably resilient under noise.
\end{abstract}

* damazz@uchicago.edu 


\section{INTRODUCTION}

One of the fundamental challenges in quantum simulation is the storage and propagation of exponentially scaling many-body quantum states. Many classical computational methods treat these states approximately, using perturbative and truncated approaches or local approximations, and result in polynomial algorithms which potentially sacrifice key characteristics of the quantum state [1, 2]. Reduced density matrix (RDM) methods focus on reducing the required state information to the $k$-body interaction inherent in the system (such as the 2-RDM for fermionic simulations) [3-15]. In these cases, the 2-RDM then is subject to its own set of criteria, such as $N$-representability, but can deal with many-body phenomena in an easier manner [3, 16 18].

An easy way to reduce the required amount of information to describe the state is through utilizing symmetries. Symmetries are conserved quantities, preserved through the preparation and propagation of a state [2, 19, 20]. For molecular systems, particle number, total and projected spin, invariance under time-reversal, and often molecular point groups are examples of symmetries. These can be used in classical electronic structure calculations to substantially reduce the number of resources necessary to simulate a system [2, 21, 22, On a quantum computer, the exponentially scaling state can be prepared efficiently for many applications. For systems with a $k$-body interaction, tomography of the $k$-RDM is sufficient to describe the system's physical properties. Storage of the state (which is critical for near-term applications) can be reduced to the tomography of the $k$-RDM based on the $k$-body interaction [15, 23 26]. In contrast to classical RDMs method, a pure quantum state would automatically satisfy the $N$-representability problem.

In quantum computing, symmetries are utilized in a wide range of settings. There is ongoing work to utilize number-preserving gate sequences [27, 28], design algorithms for preparing symmetry preserving ansatz [29 35], reduce symmetry violations through variational constraints [36 38, reduce the simulated Hilbert space through varied applications of symmetries [39 41], and to utilize symmetries as a form of error mitigation [42 44]. 
Despite potential reductions in the state complexity using symmetries or other methods, tomography still can require a large set of measurements. For molecular systems, the Hamiltonian and 2-RDM scale as $O\left(r^{4}\right)$, where $r$ is the number of basis functions, and many heuristic and systematic ways involving graph-theoretic or combinatorial approaches have been introduced to lower this number, which in some cases can render an apparent scaling of $O\left(r^{3}\right)$ or $O\left(r^{2}\right)$ with swap networks [24, 45,47]. Within quantum simulation as a whole, fermionic tomography is particularly challenging due to the nonlocal characteristics of fermionic operators and prevents a logarithmic reduction in complexity seen with other qubit systems [24].

In this work we present a method of lowering measurement and tomography costs for quantum states and RDMs by exploiting the quantum state's symmetries. By finding the symmetry projected form of our measurement operators, we can re-express our operators in a minimal basis on the quantum computer. The method leads to a constant scaling improvement in the number of terms which has to be measured, and can be combined with other measurement techniques to reduce circuit preparation costs for near-term calculations.

\section{THEORY}

A symmetry for a quantum system can be defined mathematically as a non-zero operator $\hat{S}$ which commutes with the system Hamiltonian $\hat{H}$,

$$
[\hat{H}, \hat{S}]=0
$$

Consider a set of $n$ symmetries $\mathcal{S}=\left\{S_{1}, S_{2}, \ldots, S_{n}\right\}$ where each symmetry commutes with all other symmetries (note that the most common set in fermionic simulation of $\hat{N}, \hat{S}_{z}$, and $\hat{S}^{2}$ obeys this). We can find a basis which is a mutual eigenbasis of each element of $\mathcal{S}$, and then we denote a wavefunction which obeys each of these symmetries:

$$
|\psi\rangle=\sum_{\alpha} c_{\alpha}\left|\alpha, s_{1}, s_{2}, \ldots, s_{n}\right\rangle
$$


where each $s_{i}$ represents the eigenvalues of the $i$-th symmetry. Now, let $\hat{A}$ be an operator acting on this state in this symmetry basis:

$$
\hat{A}=\sum a_{\beta, u_{1}, u_{2}, \ldots u_{n}}^{\alpha, t_{1}, t_{2}, \ldots t_{n}}\left|\alpha, t_{1}, t_{2}, \ldots, t_{n}\right\rangle\left\langle\beta, u_{1}, u_{2}, \ldots, u_{n}\right|
$$

Then, if we are interested in the expectation of $\hat{A}$, we can evaluate it as:

$$
\langle\hat{A}\rangle=\sum_{i, j} c_{i}^{*} c_{j} a_{j, s_{1}, s_{2}, \ldots, s_{n}}^{i, s_{1}, s_{2}, \ldots, s_{n}}
$$

and so we have projected $\hat{A}$ into the specific subspace of each symmetry, despite that $\hat{A}$ does not necessarily commute with $\hat{S}$. Note that if each symmetry did not commute, our eigenvectors would not be simultaneous eigenstates, and we could instead apply the operators in terms of increasing restrictions as relevant to the quantum state.

In quantum simulation, often we measure operators which likely do not violate these symmetries individually but often cannot be directly measured on the quantum computer.Instead, we map these operators to a set of measurable operators on the quantum device, which commonly today are projective measurements onto eigenvectors of the Pauli matrices. The basis of operators will not always commute with the state's symmetries, and so will be projected by the wavefunction.

To find the projected form, we could explicitly calculate the operator form in Eq. (4) for small systems, but this quickly becomes unfeasible with increasing system size. By noting that most operators we are interested in act non-trivially on a few local sites, we can find a projected form acting on the local space. The form of this operator can be found relatively easily for particular symmetries, and we derive our approach in Appendix A. Instead of focusing on one symmetry state $s$, we project our operator onto a symmetry conserving subspace:

$$
\langle\tilde{A}\rangle=\sum_{s}\left\langle\hat{P}_{s} \hat{A} \hat{P}_{s}\right\rangle=\sum_{s} \sum_{i, j} c_{i}^{*} c_{j} a_{j, s}^{i, s}
$$

where $\hat{P}_{s}$ is a projection onto a single symmetry $s$. The projected form can be viewed as a mixed operator resulting from projecting onto different pure symmetry states. Notably, 
TABLE I. Example procedure for finding a set of symmetry projected operators. The vector representation of $\hat{A}$ is given as $\vec{A}$.

Given operators sets for measurement $(M)$, symmetries $(\mathcal{S})$ and the computational basis $(A)$;

(0) Find a set of projection operators $\hat{P}_{s}$;

(1) For each measurement operator $\hat{m} \in M$ :

(a) Transform $\hat{m}$ in $A, \hat{m}=\sum_{i} a_{i} \hat{A}_{i}$

(b) For each $\hat{A}_{i}$, find symmetry projected computational operators $\hat{A}_{i}^{c}$

(c) Choose linearly independent $\vec{A}_{j}$ as columns of $U$ and solve for $U \vec{x}_{m}=\vec{m}$ (2) Apply further processing

both $\tilde{A}$ and the projected $\hat{A}$ will generally contain significantly less terms than the native qubit operators.

With these points in view, our approach is as follows. Given an operator $\hat{M}$, we express it in the Pauli basis using some transformation:

$$
\hat{M}=\sum_{i} a_{i} \hat{A}_{i}
$$

where $\hat{A}_{i}$ are typically Pauli strings. Then, we apply our symmetry projection to the individual $\hat{A}_{i}^{c}=\sum_{s} \hat{P}_{s} \hat{A}_{i} \hat{P}_{s}$. We represent both the operator and the Pauli strings in a vector form $\left(\vec{m}\right.$, and $\left.\vec{A}_{i}^{c}\right)$ and then using $\vec{A}_{i}^{c}$ as columns, form a matrix of linearly independent vectors, $U$.

Finally, we solve the linear system of equations for a vector $\vec{x}$ :

$$
U \vec{x}=\vec{m}
$$

to obtain a new basis of measurement for $\hat{M}$ which is equal to or lower in dimension. In general, this will not be unique, and we can order our selection process or bias it to affect the set of terms. The process here is summarized in Table 1 and an example is included in Appendix B. 


\section{RESULTS AND APPLICATIONS}

\section{A. Application to Reduced Density Operators}

This work's inspiration is centered on molecular and fermionic systems, which only need characterization of the particles pairwise interactions. These are completely captured in the two-electron reduced density matrix, or 2-RDM, which represents a partial tomography of the quantum state. Elements of the 2-RDM are measured according to:

$$
{ }^{2} D_{j, l}^{i, k}=\left\langle\psi\left|a_{i}^{\dagger} a_{k}^{\dagger} a_{j} a_{l}\right| \psi\right\rangle
$$

where $i, j, k$, and $l$ are spin orbital indices. On the quantum computer, the most basic mapping from fermions to qubits is the Jordan-Wigner transformation. This transforms the creation and annihilation operators as [48]:

$$
\begin{aligned}
a_{j}^{\dagger} & =\frac{1}{2}\left(X_{j}-i Y_{j}\right) \bigotimes_{k=1}^{j-1} Z_{k}, \\
a_{j} & =\frac{1}{2}\left(X_{j}+i Y_{j}\right) \bigotimes_{k=1}^{j-1} Z_{k},
\end{aligned}
$$

where $X_{j}, Y_{j}$, and $Z_{j}$ indicate a Pauli operator acting on qubit $j$. The local aspect of the operation on a qubit is defined by the $X$ and $Y$ gates, whereas the $Z$ portion generates parityconserving gates. The parity mapping [49, 50] exchanges the storage of orbital occupations and parity, and the Bravyi-Kitaev mapping stores both in a tree-like diagram [51]. Both of these schemes form linear combinations of operators which act differently on local sites and identically on nonlocal sites, and thus can be symmetry projected with our technique.

For higher order RDMs (which can be used for exploring states in a linear- or quadraticexpansive subspace or in other methods [52]), similar advantages can be seen. We show the effect of our symmetry projection technique in reducing the number of measurements for 1, 2, and 3-RDMs in Table II. We specify cases with different number of excitations because particle and hole operators commute with the given molecular symmetries, whereas

excitations (or de-excitations) will transform to operators in the computational basis that 
individually may not conserve a given symmetry. The set of measurements in performing tomography of the 2-RDM contains the set required for molecular Hamiltonians, and thus can be used in Hamiltonian based measurement. An advantage of focusing on the 2-RDM is that it enables systematic approaches in the tomography and that the 2-RDM itself can be used as a tool in error-mitigation [24, 25, 35, 52, 53].

One technique to lower measurement costs involves using the qubit-wise commutation relation, which relates Pauli strings which can be concatenated and thus simultaneously measured through local measurement schemes. Finding the optimal grouping is a NP hard problem, but by characterizing the set of measurements as a graph problem connected by this relation, we can use coloring algorithms to reduce the number of colors (or cliques) needed [47, 54]. To consider the advantage in using our projected technique, we can compare the number of cliques obtained with the default tomography to number obtained with our method. We explore this for obtaining 2-RDMs of differing sizes in Figure 1. Additionally, the worst-case scaling of the number of tomography terms is $\mathcal{O}\left(r^{4}\right)$, and we look at overall scaling coefficient $\left(r^{n}\right)$ under the grouping technique with both measurement schemes. If one used the maximally commuting method [46, 47], which for the most part finds larger groups of operators that can be simultaneously measured, the circuit depth scales polynomially with the number of terms in a group, and so our scheme would lead to reductions in the transformation required.

\section{B. Effects of Noise on Particle Count}

One implicit assumption in the above work is that the quantum state is of decent quality and that it preserves the proper symmetries throughout the simulation. Due to noise, this will almost certainly never be the case, so we are interested in how noise can affect our symmetry projected scheme's quality.

From a theoretical perspective, we can envision two broad cases. In the first, we have a 
FIG. 1. (Left) Ratio of the number of total required terms in the 2-RDM versus the number of required circuits following a grouping procedure, and (right) the scaling coefficient of the number of circuits with respect to the number of qubits. The color denotes the fermionic mapping (JordanWigner, Parity, and Bravyi-Kitaev), and the symbol denotes the set of symmetries applied in the projection procedure. The grouping procedure involves grouping terms according to qubit-wise commutation following Ref. [54]. The black line on the right refers to total number of terms. See Appendix $\mathrm{C}$ for more details.
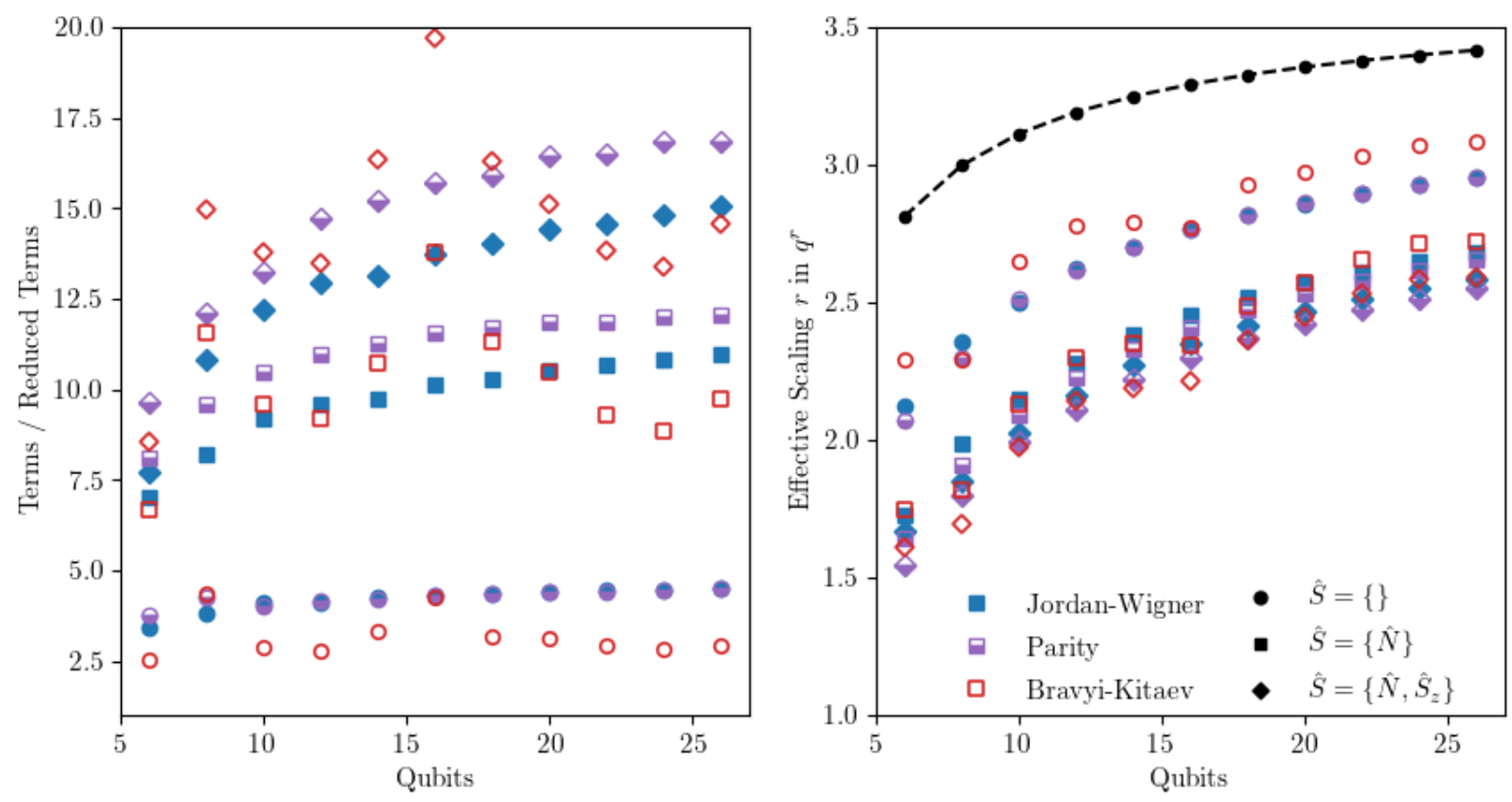

mixed state which is a sum of weighted states:

$$
\rho=\sum_{s} \sum_{i} \alpha_{s i}|i, s\rangle\langle i, s|
$$

In this case the symmetry projection is still exact, as the states are orthogonal to each other, and our method will not be affected by errors. The second case involves a state that is a mixture of different symmetry states, in which case our reduced tomography no longer represents the true tomography. Yet, whether or not standard tomography would offer significant advantages in this case is unclear, as significant errors still corrupt the system in a variety of ways.

To investigate the effects of noise, we simulated a minimal two-fermion in four-spin orbital 
TABLE II. Dimension of the number of Pauli measurements required for tomography of the 1- and 2-RDMs in the traditional (naive) and symmetry projected (reduced) approaches for given spin and spatial configurations of the second quantized measurement operators with $\hat{N}$, and $\hat{S}_{z}$ symmetries under the Jordan-Wigner transformation. We also give examples of the sets of operators in the naive $(N)$ and reduced $(R)$ methods corresponding with operators marked with a $(*)$. Note, $R$ is not unique in any of these cases. The cases including the $\hat{S}^{2}$ symmetry do not greatly affect the results, but require covering the many permutations of the spatial orbitals. A bar across spins indicates an excitation or de-excitation between these orbitals, and only the unique spin configuration is shown. $\times$ indices the Cartesian product of two sets. $\vec{Z}$ indicates a tensor product of $Z$ gates which is constant across all operations.

\begin{tabular}{|c|c|c|c|c|c|}
\hline$k-\mathrm{RDM}$ & Spin & $q$-Sites & Naive & Reduced & Example Operator and Sets of Measurement Operators \\
\hline \multirow[t]{3}{*}{1} & $\alpha \alpha$ & 1 & 2 & 2 & \multirow{3}{*}{$\begin{array}{c}a_{i}^{\dagger} a_{j}, \quad\{i, j\} \in \alpha \\
\mathrm{N}=\left\{X_{i}, Y_{i}\right\} \times\left\{X_{j}, Y_{j}\right\} \vec{Z}, \mathrm{R}=\left\{X_{i} X_{j} \vec{Z}, X_{i} Y_{j} \vec{Z}\right\}\end{array}$} \\
\hline & $\overline{\alpha \alpha}^{*}$ & 2 & 4 & 2 & \\
\hline & $\alpha \beta$ & - & - & 0 & \\
\hline \multirow[t]{7}{*}{2} & $\alpha \alpha \alpha \alpha$ & 2 & 4 & 4 & \multirow{3}{*}{$a_{i}^{\dagger} a_{k}^{\dagger} a_{l} a_{j}+a_{j}^{\dagger} a_{l}^{\dagger} a_{k} a_{i}$} \\
\hline & $\alpha \alpha \overline{\alpha \alpha}$ & 3 & 8 & 4 & \\
\hline & $\overline{\alpha \alpha \alpha \alpha}$ & 4 & 16 & 6 & \\
\hline & $\alpha \alpha \alpha \beta$ & - & - & 0 & \multirow{4}{*}{$\begin{array}{c}\mathrm{N}=\left\{X_{i} X_{k}, Y_{i} Y_{k}\right\} \times\left\{X_{l} X_{j}, Y_{l} Y_{j}\right\} \vec{Z} \cup \\
\left\{X_{i} Y_{k}, Y_{i} X_{k}\right\} \times\left\{X_{l} Y_{j}, Y_{l} X_{j}\right\} \vec{Z} \\
\mathrm{R}=\left\{X_{i} X_{k} X_{l} X_{j} \vec{Z}, X_{i} Y_{k} Y_{l} X_{\mathrm{j}} \vec{Z}\right\}\end{array}$} \\
\hline & $\alpha \alpha \beta \beta$ & 2 & 4 & 4 & \\
\hline & $\alpha \alpha \overline{\beta \beta}$ & 3 & 8 & 4 & \\
\hline & $\overline{\alpha \alpha} \bar{\beta} \beta^{*}$ & 4 & 16 & 4 & \\
\hline \multirow[t]{12}{*}{3} & $\alpha \alpha \alpha \alpha \alpha \alpha$ & 3 & 8 & 8 & \multirow{12}{*}{$\begin{array}{c}\{l, \jmath, k, l\} \in \alpha,\{m, n\} \in \beta \\
\mathrm{N}=\left\{X_{i} X_{k}, Y_{i} Y_{k}\right\} \vec{Z} \times\left(\left\{X_{m} X_{n}, Y_{m} Y_{n}\right\} \times\left\{X_{l} X_{j}, Y_{l} Y_{j}\right\} \cup\right. \\
\left.\left\{X_{m} Y_{n}, Y_{m} X_{n}\right\} \times\left\{X_{l} Y_{j}, Y_{l} X_{j}\right\}\right) \cup \\
\left\{X_{i} Y_{k}, Y_{i} X_{k}\right\} \vec{Z} \times\left(\left\{X_{m} Y_{k}, Y_{m} X_{n}\right\} \times\left\{X_{l} X_{j}, Y_{l} Y_{j}\right\} \cup\right. \\
\left.\left\{X_{m} X_{n}, Y_{m} Y_{n}\right\} \times\left\{X_{l} Y_{j}, Y_{l} X_{j}\right\}\right), \\
\mathrm{R}=\left\{\left\{\left(X_{i} X_{k} Y_{l} X_{j}, Y_{i} X_{k} X_{l} X_{j}, X_{i} Y_{k} X_{l} X_{j}\right\} \times Y_{m} X_{n} \vec{Z} \cup\right.\right. \\
\left.\left\{X_{i} X_{k} X_{l} X_{j}, Y_{i} Y_{k} X_{l} X_{j}, Y_{i} X_{k} Y_{l} X_{j}\right\} \times X_{m} X_{n} \vec{Z}\right\}\end{array}$} \\
\hline & $\alpha \alpha \alpha \alpha \overline{\alpha \alpha}$ & 4 & 16 & 8 & \\
\hline & $\alpha \alpha \overline{\alpha \alpha \alpha \bar{\alpha}}$ & 5 & 32 & 12 & \\
\hline & $\alpha \alpha \alpha \alpha \alpha \alpha$ & 6 & 64 & 20 & \\
\hline & $\alpha \alpha \alpha \alpha \alpha \beta$ & - & - & 0 & \\
\hline & $\alpha \alpha \alpha \alpha \beta \beta$ & 3 & 8 & 8 & \\
\hline & $\alpha \alpha \overline{\alpha \alpha} \beta \beta$ & 4 & 16 & 8 & \\
\hline & $\alpha \alpha \alpha \alpha \bar{\beta} \beta$ & 4 & 16 & 8 & \\
\hline & $\alpha \alpha \overline{\alpha \alpha} \bar{\beta} \beta$ & 5 & 32 & 8 & \\
\hline & $\overline{\alpha \alpha \alpha \alpha \beta \beta}$ & 5 & 32 & 12 & \\
\hline & $\overline{\alpha \alpha \alpha \alpha \beta \beta^{*}}$ & 6 & 64 & 12 & \\
\hline & $\alpha \alpha \alpha \beta \beta \beta$ & - & - & 0 & \\
\hline
\end{tabular}


system on a quantum device and with an accompanying noise model. The results are seen in Table III. Importantly, the distance between the RDMs produced by the tomography methods is comparable to statistical noise, and is always less than then the distance from the true 2-RDM, when noise is present. In the case of the noise-free result, the larger variance between the two tomography methods is likely a result of propagation of sampling error, which is absent with the ideal 2-RDM.

TABLE III. A comparison of 2-RDMs of a two-electron system under varying levels of simulated noise (simulated and experimental) through the Frobenius norm of the difference matrices at randomly sampled points. ${ }^{2} D$ refers to the ideal 2 -RDM, ${ }^{2} \tilde{D}$ refers to standard tomography of the 2-RDM under a noise model, and ${ }^{2} \tilde{D}^{c}$ refers to the 2-RDM constructed from symmetry projected tomography under a noise model. Values represent averages of the Frobenius norms of difference matrices over 25 random states of $\mathrm{H}_{2}$ in a minimal basis where the ansatz includes 3 parameters. In general, the differences between the noisy tomography methods are consistently much smaller than the difference to the ideal state, and are almost indistinguishable from stochastic effects (seen at the $n=\infty$ limit). More details regarding these results are included in Appendix C.

\begin{tabular}{c|c|c|c}
\hline$\Delta=$ & ${ }^{2} D-{ }^{2} \tilde{D}$ & ${ }^{2} D-{ }^{2} \tilde{D}^{c}$ & ${ }^{2} \tilde{D}-{ }^{2} \tilde{D}^{c}$ \\
\hline Noise Strength, $\left(\frac{1}{2}\right)^{n}$ & $\|\Delta\|_{F}$ & $\|\Delta\|_{F}$ & $\|\Delta\|_{F}$ \\
\hline$n=0$ & $0.68(6)$ & $0.69(5)$ & $0.05(1)$ \\
$n=1$ & $0.39(4)$ & $0.39(4)$ & $0.049(9)$ \\
$n=2$ & $0.22(2)$ & $0.23(3)$ & $0.05(1)$ \\
$n=3$ & $0.17(1)$ & $0.17(2)$ & $0.046(9)$ \\
$n=4$ & $0.13(1)$ & $0.14(1)$ & $0.05(1)$ \\
$n=\infty$ & $0.027(5)$ & $0.036(8)$ & $0.05(1)$ \\
\hline Experimental & $0.87(5)$ & $0.87(4)$ & $0.05(1)$ \\
\hline
\end{tabular}




\section{CONCLUSION}

Modern quantum computing has advanced drastically in the past decade, with a surge of incremental improvements in experimental and algorithmic improvements. Circuit optimization, qubit reduction, reducing the required parameter space in the classical optimization, or lowering tomography and measurement costs all have attempted to capitalize on the available quantum resources maximally. Work in utilizing system symmetries explores a fascinating aspect of quantum mechanics, and we hope that future work will continue to apply these ideas in lowering costs.

Our approach in utilizing symmetry projected operators provides a simple way to reduce the number of measurements needed, and when combined with other techniques can lead to large reductions in the effective scaling of the system. The routine can be performed in one step before the calculation, and adds no additional cost to the quantum or classical algorithm.

\section{ACKNOWLEDGEMENTS}

D.A.M. gratefully acknowledges the Department of Energy, Office of Basic Energy Sciences, Grant DE-SC0019215 and the U.S. National Science Foundation Grants No. CHE2035876 and No. DMR-2037783.

\section{DATA AVAILABILITY}

Data is available from the corresponding author upon reasonable request.

\section{Appendix A: Derivation of Local Symmetry Operators}

In Section II we noted we would like to use a symmetry which exists on the local subspace

of the system instead of the entire system for our method. While in general this will not 
hold for every symmetry, we discuss ways to identify and utilize these symmetries here.

If we consider a state as being in an eigenstate $s$ of a symmetry $\hat{S}$, as seen in Eq. (5) the projection of the state of $s$ onto an operator $A$ is:

$$
\left\langle P_{s} \hat{A} P_{s}\right\rangle=\sum_{i, j} c_{i, s} c_{j, s}^{*} a_{j, s}^{i, s}
$$

Let the state exist in the Fock space of $M$ orbitals, $\mathcal{F}(M)$. Assume that we have symmetry operators $\hat{T}_{1}$ and $\hat{T}_{2}$ which act on subspaces $F_{1}=\mathcal{F}(N)$ and $F_{2}=\mathcal{F}(M-N)$ respectively, and that we can express an eigenstate of $\hat{S}$ as:

$$
|i, s\rangle=\sum_{\alpha, \beta} \sum_{t} R_{s t t^{\prime}}^{i \alpha \beta}|\alpha, t\rangle \otimes\left|\beta, t^{\prime}\right\rangle
$$

where $t$ is an eigenstate of $\hat{T}_{1}, t^{\prime}$ is an eigenstate on $\hat{T}_{2}$ such that the direct product of vectors will be eigenvectors of the symmetry value $s$, and $R$ is a rank-three tensor providing an index between the total space and the two smaller subspaces. We can also imagine a reduced representation, where we omit some of the exact state information, considering only blocks in different symmetries (as the orthogonality of the states is not used here):

$$
|s\rangle=\sum_{t} R_{t, t^{\prime}}^{s}|t\rangle \otimes\left|t^{\prime}\right\rangle
$$

. Now, let $\hat{A}$ be an operator which acts primarily on $F_{1}$ and only adds a phase onto elements of $F_{2}$, which in denoted as:

$$
\hat{A}=\sum_{a, b, c} A_{a, b, c}|a\rangle\langle b|\otimes| c\rangle\langle c|
$$

where $A$ is also a rank-three tensor, indicating the phase for a given state in $F_{2}$. For nonfermionic operators this would is unity across all states, whereas for fermionic operators there will be some phase changes depending on the states $\gamma$. Using this to evaluate the 
expectation of $\hat{A}$ we find:

$$
\begin{aligned}
\operatorname{Tr} \hat{A} \rho= & \operatorname{Tr} \sum_{i, j} \sum_{\alpha, \beta, \gamma, \delta, \epsilon, \zeta, \eta} \sum_{a, b, c, d, e} c_{i, s} c_{j, s}^{*} A_{a, b, c}^{\alpha, \beta, \gamma} R_{s, d, d^{\prime}}^{i, \delta, \epsilon} R_{s, e, e^{\prime}}^{* j, \zeta, \eta}|\alpha, a\rangle\langle\beta, b \mid \delta, d\rangle\langle\zeta, e| \\
& \otimes|\gamma, c\rangle\left\langle\gamma, c \mid \epsilon, d^{\prime}\right\rangle\left\langle\eta, e^{\prime}\right| \\
= & \operatorname{Tr}_{1} \sum_{i, j} \sum_{\alpha, \beta, \gamma, \delta, \zeta} \sum_{a, b, c} c_{i, s} c_{j, s}^{*} A_{a b c}^{\alpha \beta \gamma} R_{s c^{\prime} c}^{i \delta \gamma} R_{s c^{\prime} c}^{* j \zeta \gamma}|\alpha, a\rangle\left\langle\beta, b \mid \delta, c^{\prime}\right\rangle\left\langle\zeta, c^{\prime}\right| \\
= & \sum_{k} \operatorname{Tr}_{1} \sum_{i, j} \sum_{\alpha, \beta, \gamma, \delta, \zeta} c_{i, s} c_{j, s}^{*} A_{k k k^{\prime}}^{\alpha \beta \gamma} R_{s k k^{\prime}}^{i \delta \gamma} R_{s k k^{\prime}}^{* j \zeta \gamma}|\alpha, k\rangle\langle\beta, k \mid \delta, k\rangle\langle\zeta, k| .
\end{aligned}
$$

In the last two steps we used the fact that following the partial trace over $F_{2}$ the total spin of the system must still give $s$, yielding a mixed state of pure symmetry states of $\hat{T}_{1}$. Through linearity we can extract a projection operator and apply it to elements of $\hat{A}$, which leads to a greatly simplified form of the operator. This is equvialent to Eq. (5) in the main text, and this projected form can be found for our set of transformed measurement opeartors.

The symmetries discussed in the text relating to molecular systems, $\hat{N}, \hat{S}_{z}$, and $\hat{S}^{2}$, all can be described in this way, and thus can be applied using this method. Symmetries which are elements of the Pauli group (i.e. tensored products of Pauli matrices) also satisfy these conditions.

\section{Appendix B: Examples of Symmetry Projection}

To illustrate our method, let $|\psi\rangle$ be a two-electron system in four $\alpha$-spin orbitals and $\hat{M}$ be defined as:

$$
\hat{M}=c_{1} a_{1}^{\dagger} a_{2}+c_{2} a_{2}^{\dagger} a_{1} .
$$

$\hat{M}$ here is a linear combination of $1-\mathrm{RDM}$ operators. The system as a whole will obey the number symmetry $\hat{N}=\sum_{i=1}^{4} a_{i}^{\dagger} a_{i}$, but we can apply a reduced symmetry $\hat{N}_{1}=\sum_{i=1}^{2} a_{i}^{\dagger} a_{i}$ to our operator. The reduced symmetry operator will have projections operators onto the $N=0, N=1$, and $N=2$ subspaces, which can be written as:

$$
P_{0}=|00\rangle\left\langle 00\left|, P_{1}=\right| 01\right\rangle\langle 01|+| 10\rangle\left\langle 10\left|, P_{2}=\right| 11\right\rangle\langle 11| .
$$


Using the Jordan-Wigner transformation we can express $\hat{M}$ in terms of Pauli matrices yielding:

$$
\hat{M}=\frac{1}{4}\left(c_{1}+c_{2}\right)\left(X_{1} X_{2}+Y_{1} Y_{2}\right)+\frac{i}{4}\left(c_{1}-c_{2}\right)\left(X_{1} Y_{2}-Y_{1} X_{2}\right)=\left(\begin{array}{cccc}
0 & 0 & 0 & 0 \\
0 & 0 & c_{2} & 0 \\
0 & c_{1} & 0 & 0 \\
0 & 0 & 0 & 0
\end{array}\right) .
$$

One of the operators in the Pauli basis, $A_{X X}$, can be projected as follows:

$$
\hat{A}_{X X}^{c}=\sum_{i} P_{i}\left(X_{1} X_{2}\right) P_{i}=\left(\begin{array}{llll}
0 & 0 & 0 & 0 \\
0 & 0 & 1 & 0 \\
0 & 1 & 0 & 0 \\
0 & 0 & 0 & 0
\end{array}\right)
$$

To express this as a vector in the computational basis in the operator space, let $e_{i, j}$ denote a basis element where $i, j$ denote the row and column index, which gives:

$$
\vec{A}_{X X}=e_{10,01}+e_{01,10}
$$

Similar vector forms can be found for the other Pauli matrices:

$$
\begin{aligned}
& \vec{A}_{X Y}^{c}=-i e_{10,01}+i e_{01,10} \\
& \vec{A}_{Y X}^{c}=i e_{10,01}-i e_{01,10} \\
& \vec{A}_{Y Y}^{c}=e_{10,01}+e_{01,10} .
\end{aligned}
$$

Clearly, we are limited by the dimension of the span of these vectors, leaving us to choose two vectors. Using $\vec{A}_{X X}^{c}$ and $\vec{A}_{X Y}^{c}$ as the column vectors of $U$, and $\vec{m}$ as the target vector, we can find the solution to the system of linear equations $U \vec{x}=\vec{m}$ :

$$
\left(\begin{array}{cc}
1 & -i \\
1 & i
\end{array}\right)\left(\begin{array}{l}
x_{1} \\
x_{2}
\end{array}\right)=\left(\begin{array}{l}
c_{1} \\
c_{2}
\end{array}\right) \rightarrow \vec{x}=\frac{1}{2}\left(\begin{array}{c}
c_{1}+c_{2} \\
i c_{1}-i c_{2}
\end{array}\right)
$$

Thus a measurement of the form:

$$
\hat{X}=\frac{c_{1}+c_{2}}{2} X_{1} X_{2}+\frac{i\left(c_{1}-c_{2}\right)}{2} X_{1} Y_{2}
$$

yields equivalent information as the traditional measurement due to symmetries of the system. 


\section{Appendix C: Computational Details}

Figure 1 was created by first generating the set of 2-RDM operators for a given molecular system and transforming them into a set of Pauli operators using fermionic-to-qubit transformations followed by our symmetry projection technique. To efficiently group terms, we expressed the set of operators as a graph utilizing the qubit-wise commuting relationship, and used an algorithm to attempt to find the minimimum clique cover [54]. The graphs were stored using the graph-tools (v 2.29) Python package [55]. A sequential coloring algorithm was used where we selected the vertices with the largest number of edges first, as this proved to be a reliable approach which was scalable to larger qubit systems which did not yield significantly worse results than the related recursive method [54].

Table 3 was generated with the help of Qiskit (v 0.15.0) [56], and simulates a twoelectron system in a minimal basis (STO-3G) under the Jordan-Wigner transformation on four qubits. We prepared 25 random states parameterized by a double excitation and two single excitations. The experimental results were obtained on the IBMQ Bogota device. The noise model was based on the backend-centered model in the Aer module of Qiskit, which approximates the noise channels mainly as a product of depolarizing and thermal relaxation channels acting locally on single- and two-qubit gates. The parameters were based on averages of the experimental devices, and were scaled down according to $\tau$ to model a decrease in the strength of the noise.

\section{Appendix D: Further Computational Details}

For the quantum computation and noise simulation in Table III of the main text, we used the quantum computer IBMQ Bogota (5-qubits) provided through the IBM Quantum Experience, as well as a noise model based on the device. The quantum device has fixed-frequency transmon qubits with co-planer waveguide resonators [57, 58]. The Python package Qiskit (v 0.15.0) [56] was used to interface with the device. Device properties can be found in Supplemental Table IV. 
Supplemental Table IV. Calibration data for the ibm-bogota device taken on November $2^{\text {nd }}$, 2020, from benchmarking. $\mathrm{U}_{2}$ and $\mathrm{U}_{3}$ represent single qubit gate errors containing one and two $X_{\pi / 2}$ pulses and two and three frame changes respectively. $\mathrm{RO}_{i \mid j}$ represents the probability of measuring the state $i$ given a prepared state $j . T_{1}$ and $T_{2}$ are the given thermal relaxation times for each qubit. Frequency refers to the qubits opeartional frequency, and influences the excited state population based on the device temperature. $[j]$ specifies the target qubit with control qubit $i$, and the number in paranthesis after each entry in the CNOT column indicates the gate length. The gate lengths for the $U_{2}$ and $U_{3}$ gates were $35 \mathrm{~ns}$ and 71 ns respectively.

\begin{tabular}{|c|c|c|c|c|c|c|c|c|}
\hline $\begin{array}{c}\text { Qubit } \\
i\end{array}$ & $\begin{array}{c}\text { Frequency } \\
\mathrm{GHz}\end{array}$ & $\begin{array}{c}\mathbf{U}_{2} \\
\left(10^{-4}\right)\end{array}$ & $\begin{array}{c}\mathbf{U}_{3} \\
\left(10^{-4}\right)\end{array}$ & $\begin{array}{l}\mathbf{R O}_{0 \mid 1} \\
\left(10^{-2}\right)\end{array}$ & $\begin{array}{l}\mathbf{R O}_{1 \mid 0} \\
\left(10^{-2}\right)\end{array}$ & $\begin{array}{c}\mathbf{T}_{1} \\
(\mu s)\end{array}$ & $\begin{array}{r}\mathbf{T}_{2} \\
(\mu s)\end{array}$ & $\begin{array}{c}{[j] \mathbf{C N O T}_{i}^{j}} \\
\left(10^{-2}\right)\end{array}$ \\
\hline 0 & 5.000 & 3.7 & 4.5 & 3.6 & 8.0 & 93.6 & 133.3 & [1] $2.0(690)$ \\
\hline 1 & 4.8 & 3.2 & 6 & 17.3 & 15.1 & 59.9 & 58.5 & {$[0] 2.0(654)[2] 1.0(498)$} \\
\hline 2 & 4.783 & 1.7 & 3.3 & 5.7 & 3.6 & 77.7 & 120.6 & [1] $1.0(533)[3] 1.0(626)$ \\
\hline 3 & 4.858 & 2.4 & 4.8 & 3.0 & 0.9 & 131.1 & 187.1 & {$[2] 1.0(590)[4] 4.8(370)$} \\
\hline 4 & 4.978 & 13.8 & 27.5 & 5.2 & 2.6 & 101.7 & & [3] $4.8(334)$ \\
\hline
\end{tabular}

The circuit used is based on the Jordan-Wigner transformation and uses exponentials of anti-Hermitian operators. Because of the size of the system and the indistinguishablity of the action of the different Pauli operators on the state, we can use a single term to describe each of the relevant excitations [35, 43]. The target circuit can be written as:

$U=\left[\exp \theta_{1}\left(a_{\alpha 0}^{\dagger} a_{\alpha 1} a_{\beta 0}^{\dagger} a_{\beta 1}-a_{\beta 1}^{\dagger} a_{\beta 0} a_{\alpha 1}^{\dagger} a_{\alpha 0}\right)\right]\left[\exp \theta_{2}\left(a_{\alpha 0}^{\dagger} a_{\alpha 1}-a_{\alpha 1}^{\dagger} a_{\alpha 0}\right)\right]\left[\exp \left(\theta_{3} a_{\alpha 0}^{\dagger} a_{\alpha 1}-a_{\alpha 1}^{\dagger} a_{\alpha 0}\right)\right]$

which can be simulated with limited Pauli terms as:

$$
U^{\prime}=\left[\exp i \theta_{1} Y_{1} X_{2} X_{3} X_{4}\right]\left[\exp i \theta_{2} Y_{1} X_{2}\right]\left[\exp i \theta_{3} Y_{3} X_{4}\right]
$$

and then simplified according to normal procedures. Our resulting circuit had 8 CNOT gates and 9 single qubit gates prior to measurement. We performed tomography of both the real and imaginary elements of the 2-RDM despite having only a real wavefunction. The list of measurement circuits generated for the normal circuit (following the grouping procedure) 


$$
\begin{aligned}
N=\{ & Y_{1} X_{2} Z_{3} Z_{4}, X_{1} X_{2} Z_{3} Z_{4}, Y_{1} Y_{2} Z_{3} Z_{4}, X_{1} Y_{2} Z_{3} Z_{4}, Z_{1} Z_{2} Y_{3} X_{4}, \\
& Z_{1} Z_{2} X_{3} X_{4}, Z_{1} Z_{2} Y_{3} Y_{4}, Z_{1} Z_{2} X_{3} Y_{4}, Y_{1} X_{2} Y_{3} X_{4}, X_{1} X_{2} Y_{3} X_{4}, \\
& Y_{1} Y_{2} Y_{3} X_{4}, X_{1} Y_{2} Y_{3} X_{4}, Y_{1} X_{2} X_{3} X_{4}, X_{1} X_{2} X_{3} X_{4}, Y_{1} Y_{2} X_{3} X_{4}, \\
& X_{1} Y_{2} X_{3} X_{4}, Y_{1} X_{2} Y_{3} Y_{4}, X_{1} X_{2} Y_{3} Y_{4}, Y_{1} Y_{2} Y_{3} Y_{4}, X_{1} Y_{2} Y_{3} Y_{4} \\
& \left.Y_{1} X_{2} X_{3} Y_{4}, X_{1} X_{2} X_{3} Y_{4}, Y_{1} Y_{2} X_{3} Y_{4}, X_{1} Y_{2} X_{3} Y_{4}, Z_{1} Z_{2} Z_{3} Z_{4}\right\}
\end{aligned}
$$

whereas the set of reduced circuits (with the same grouping procedure) is given by:

$$
\begin{aligned}
R=\{ & X_{1} X_{2} Z_{3} Z_{4}, Y_{1} X_{2} Z_{3} Z_{4}, Z_{1} Z_{2} X_{3} X_{4}, Z_{1} Z_{2} Y_{3} X_{4}, X_{1} X_{2} X_{3} X_{4}, \\
& \left.X_{1} X_{2} Y_{3} X_{4}, Y_{1} X_{2} X_{3} X_{4}, Y_{1} X_{2} Y_{3} X_{4}, Z_{1} Z_{2} Z_{3} Z_{4}\right\}
\end{aligned}
$$

\section{Appendix E: Noise Model}

The noise model used in generating the results in Table III is adapted from the provided noise model in Qiskit [56], and consists of a depolarizing channel followed by a thermal relaxation channel on each gate, with a readout error applied at measurement. Information on the model is adapted from the documentation provided in Qiskit [56]. Wood et al. is also referenced in the documentation and contains useful information regarding error channel representations and transformations [59]. A useful online discussion of the benefits (applicability to short $T_{1}$ processes such as on single qubit gates) and limitations (non- $T_{1}$ dominated behavior of CNOT gates, does not treat cross-talk errors, etc.) of the noise model is included in the references [60].

In our adaptation of the model, we averaged over all qubits for many of the parameters to reduce inconsistencies across the simulated device, and then scaled these parameters to simulate a consistently decreasing noise. We found that using either our model, the given model, or a model based solely on depolarizing noise did not result in signficant differences in the Frobenius norms of the difference matrices between the obtained 2-RDMs. 


\section{Thermal Relaxation Channel}

The $T_{1}$ time describes the thermal relaxation of the qubit from the excited state to the ground state and the $T_{2}$ time describes the coherence time of the qubit. Their respective relaxation rates for a given gate length $T_{g}$ are given as $r_{T_{1}}=\exp \frac{-T_{g}}{T_{1}}$ and $r_{T_{2}}=\exp \frac{-T_{g}}{T_{2}}$. For $T_{2}<T_{1}, T_{1}$ relaxation becomes the main consideration and the channel is expressed as a mixture of reset operations (proejctive measurements to $|0\rangle$ or $|1\rangle$ ) and unitary errors. Consider a ground state population $n_{0}$ and an excited state population:

$$
n_{1}=\left(1+\exp \frac{2 h f}{k_{B} T}\right)^{-1}
$$

where $h$ is Planck's constant, $k_{B}$ is the Boltzmann constant, $T$ and $f$ are the qubit temperature and frequency, and $n_{0}+n_{1}=1$. The probability of a reset error occuring is defined as:

$$
p_{\text {reset }}=1-r_{T_{1}} \text {. }
$$

Using the populations we can obtain the probability to reset to the $|0\rangle$ state $p_{r 0}$, the probability to reset to the $|1\rangle$ state $p_{r 1}$, and the probability to apply a $Z$ gate $p_{z}$ as:

$$
p_{r 0}=n_{0} p_{\text {reset }}, \quad p_{r 1}=n_{1} p_{\text {reset }}, \quad p_{z}=\frac{1}{2}\left(1-p_{\text {reset }}\right)\left(1-\frac{r_{T_{2}}}{r_{T_{1}}}\right) .
$$

For the case where $T_{2}>T_{1}$, the Choi-matrix representation is used. For a nosie channel $\mathcal{E}$, the Choi-matrix in the column representation $\mathcal{C}$ is defined by:

$$
\mathcal{C}=\sum_{i, j}|i\rangle\langle j| \otimes \mathcal{E}(|i\rangle\langle j|),
$$

and the resulting action on the state can be determined as

$$
\mathcal{E}(\rho)=\operatorname{Tr}_{1} \mathcal{C}\left(\rho^{T} \otimes \mathbb{I}\right)
$$

where we trace over the first system. The Choi matrix used in the model is:

$$
\mathcal{C}=\left(\begin{array}{cccc}
1-n_{1} p_{\text {reset }} & 0 & 0 & r_{T_{2}} \\
0 & p_{e} p_{\text {reset }} & 0 & 0 \\
0 & 0 & n_{0} p_{\text {reset }} & 0 \\
r_{T_{2}} & 0 & 0 & 1-n_{0} p_{\text {reset }}
\end{array}\right)
$$


The Choi matrix is then converted to Kraus operators and then to Pauli gates to be implemented in the simulation.

\section{Depolarizing Channel}

The depolarizing channel acting on $n$ qubits is given as [61]:

$$
\mathcal{E}_{\text {depol }}=(1-\lambda) I+\lambda D
$$

where $I$ is the identity channel, $D$ is the completely depolarizing channel, and $0 \leq \lambda \leq \frac{4^{n}}{4^{n}-1}$ (for $n$ qubits) indicates the relative strength.

If we consider the total fidelity as a function of the thermal relaxation and depolarizing channels, we have that:

$$
\begin{aligned}
F & =F\left(\mathcal{E}_{\text {depol }} \circ \mathcal{E}_{\text {relax }}\right) \\
& =(1-\lambda) F\left(\mathcal{E}_{\text {relax }}\right)+\lambda F\left(D \circ \mathcal{E}_{\text {relax }}\right) \\
& =(1-\lambda) F\left(\mathcal{E}_{\text {relax }}\right)+\lambda F(D) \\
& =F\left(\mathcal{E}_{\text {relax }}\right)-\lambda \frac{F\left(\mathcal{E}_{\text {relax }}\right) d-1}{d}
\end{aligned}
$$

where $d$ is the dimension of the system and the average fidelity of the depolarizing channel is $1 / d$. From this we can solve for $\lambda$ :

$$
\lambda=d \frac{F\left(\mathcal{E}_{\text {relax }}\right)-F}{F\left(\mathcal{E}_{\text {relax }}\right) d-1} .
$$

The gate fidelity of these channels is given by:

$$
\begin{aligned}
F_{\text {avg }}(\mathcal{E}, U) & =\int d \psi\left\langle\psi\left|U^{\dagger} \mathcal{E}(|\psi\rangle\langle\psi|) U\right| \psi\right\rangle \\
& =\frac{F_{\mathrm{pro}}(\mathcal{E}, U) d+1}{d+1} \\
& =\frac{\operatorname{Tr}\left(S_{U}^{\dagger} S_{\mathcal{E}}^{\dagger}\right)+d}{d(d+1)}
\end{aligned}
$$

where $F_{\text {pro }}$ indicates the process fidelity and $S$ represents the superator representation of a quantum channel. With all of these components, the model applies the depolarizing channel onto the one- or two-qubit gate followed by the thermal relaxation channel applied to each individual qubit. 


\section{Readout Error}

Finally, the readout errors on the devices are treated as single qubit errors. These modify the output based on the probability of reading one output given another. For instance, measuring the state for the qubit $q|0\rangle$ will give $|1\rangle$ with a probability $p=\mathbf{R O}_{1 \mid 0}(q)$, and will give $|0\rangle$ with a probability $1-p$.

[1] A. Szabo and N. S. Ostlund, Modern Quantum Chemistry: Introduction to Advanced Electronic Structure Theory (Dover Publications, New York, 1996).

[2] Trygve Helgaker, Poul Jørgensen, and Jeppe Olsen, Molecular Electronic-Structure Theory (John Wiley \& Sons, Ltd, Chichester, UK, 2000) p. 908.

[3] A.J. Coleman and V.I. Yukalov, Reduced Density Matrices: Coulson's Challenge (Springer, Berlin Heidelberg New York, 2000).

[4] David A. Mazziotti, ed., Advances in Chemical Physics, Advances in Chemical Physics, Vol. 134 (John Wiley \& Sons, Inc., Hoboken, NJ, USA, 2007) p. 574.

[5] Zhengji Zhao, Bastiaan J. Braams, Mituhiro Fukuda, Michael L. Overton, and Jerome K. Percus, "The reduced density matrix method for electronic structure calculations and the role of three-index representability conditions," J. Chem. Phys. 120, 2095-2104 (2004).

[6] D. A. Mazziotti, "Realization of quantum chemistry without wave functions through first-order semidefinite programming," Phys. Rev. Lett. 93, 213001 (2004).

[7] Neil Shenvi and Artur F. Izmaylov, "Active-Space N-representability constraints for variational two-particle reduced density matrix calculations," Phys. Rev. Lett. 105, 213003 (2010).

[8] D. A. Mazziotti, "Large-scale semidefinite programming for many-electron quantum mechanics," Phys. Rev. Lett. 106, 083001 (2011).

[9] Brecht Verstichel, Helen van Aggelen, Ward Poelmans, and Dimitri Van Neck, "Variational two-particle density matrix calculation for the hubbard model below half filling using spinadapted lifting conditions," Phys. Rev. Lett. 108, 213001 (2012).

[10] C. Schilling, D. Gross, and M. Christandl, "Pinning of fermionic occupation numbers," Phys. Rev. Lett. 110, 040404 (2013). 
[11] D. A. Mazziotti, "Enhanced constraints for accurate lower bounds on many-electron quantum energies from variational two-electron reduced density matrix theory," Phys. Rev. Lett. 117, 153001 (2016)

[12] M. Piris, "Global method for electron correlation," Phys. Rev. Lett. 119, 063002 (2017).

[13] A. Rubio-García, J. Dukelsky, D. R. Alcoba, P. Capuzzi, O. B. Oña, E. Ríos, A. Torre, and L. Lain, "Variational reduced density matrix method in the doubly-occupied configuration interaction space using four-particleN-representability conditions: Application to the XXZ model of quantum magnetism," J. Chem. Phys. 151, 154104 (2019).

[14] D. A. Mazziotti, "Anti-Hermitian contracted Schrödinger equation: Direct determination of the two-electron reduced density matrices of many-electron molecules," Phys. Rev. Lett. 97, $143002(2006)$.

[15] S. E. Smart and D. A. Mazziotti, "Quantum solver of contracted eigenvalue equations for scalable molecular simulations on quantum computing devices," http://arxiv.org/abs/2004.11416v1.

[16] A. J. Coleman, "Structure of fermion density matrices," Rev. Mod. Phys. 35, 668-686 (1963).

[17] David A. Mazziotti, "Structure of Fermionic Density Matrices: Complete N-Representability Conditions," Phys. Rev. Lett. 108, 263002 (2012), arXiv:1112.5866.

[18] Jan-Niklas Boyn, Jiaze Xie, John S. Anderson, and David A. Mazziotti, "Entangled electrons drive a non-superexchange mechanism in a cobalt quinoid dimer complex," J. Phys. Chem. Lett. , 4584-4590 (2020).

[19] Richard P. (Richard Phillips) Feynman, Robert B. Leighton, and Matthew L. (Matthew Linzee) Sands, The Feynman lectures on physics $(1963-1965)$ pp. xii +513 , three volumes.

[20] David M. Bishop, Group Theory and Chemistry (Dover Publications, Mineola, N.Y., 1993).

[21] Gergely Gidofalvi and David A Mazziotti, "Spin and symmetry adaptation of the variational two-electron reduced-density-matrix method," Phys. Rev. A 72, 052505 (2005).

[22] Hiroshi Nakatsuji, "Cluster expansion of the wavefunction. Electron correlations in ground and excited states by SAC (symmetry-adapted-cluster) and SAC CI theories," Chemical Physics Letters 67, 329-333 (1979).

[23] Nicholas C. Rubin, Ryan Babbush, and Jarrod McClean, "Application of fermionic marginal constraints to hybrid quantum algorithms," New J. Phys. 20, 053020 (2018), arXiv:1801.03524. 
[24] Xavier Bonet-Monroig, Ryan Babbush, and Thomas E O'Brien, "Nearly Optimal Measurement Scheduling for Partial Tomography of Quantum States," , 1-9 (2019), arXiv:1908.05628.

[25] Scott E Smart and David A Mazziotti, "Quantum-classical hybrid algorithm using an errormitigating N-representability condition to compute the Mott metal-insulator transition," Phys. Rev. A 100, 022517 (2019).

[26] LeeAnn M. Sager, Scott E. Smart, and David A. Mazziotti, "Preparation of an exciton condensate of photons on a 53-qubit quantum computer," http://arxiv.org/abs/2004.13868v1.

[27] Marco Roth, Marc Ganzhorn, Nikolaj Moll, Stefan Filipp, Gian Salis, and Sebastian Schmidt, "Analysis of a parametrically driven exchange-type gate and a two-photon excitation gate between superconducting qubits," Physical Review A 96, 1-10 (2017), arXiv:arXiv:1708.02090v1.

[28] M. Ganzhorn, P. Egger, D. J. an d Barkoutsos, P. Ollitrault, G. Salis, N. Moll, M. Roth, A. Fuhrer, P. Mueller, S. Woerner, I. Tavernelli, and S. Filipp, "Gate-Efficient Simulation of Molecular Eigenstates on a Quantum Computer," Physical Review Applied 11, 1 (2019), arXiv:1809.05057.

[29] Hefeng Wang, S. Ashhab, and Franco Nori, "Efficient quantum algorithm for preparing molecular-system-like states on a quantum computer," Physical Review A - Atomic, Molecular, and Optical Physics 79 (2009), 10.1103/PhysRevA.79.042335, arXiv:0902.1419.

[30] James Daniel Whitfield, "Communication: Spin-free quantum computational simulations and symmetry adapted states," Journal of Chemical Physics 139 (2013), 10.1063/1.4812566.

[31] Panagiotis Kl Barkoutsos, Jerome F. Gonthier, Igor Sokolov, Nikolaj Moll, Gian Salis, Andreas Fuhrer, Marc Ganzhorn, Daniel J. Egger, Matthias Troyer, Antonio Mezzacapo, Stefan Filipp, and Ivano Tavernelli, "Quantum algorithms for electronic structure calculations: Particle-hole Hamiltonian and optimized wave-function expansions," Physical Review A 98, 022322 (2018), arXiv:1805.04340.

[32] Sean A. Fischer and Daniel Gunlycke, "Symmetry Configuration Mapping for Representing Quantum Systems on Quantum Computers," (2019), arXiv:1907.01493

[33] George S Barron, Bryan T Gard, Orien J Altman, Nicholas J Mayhall, Edwin Barnes, Sophia E Economou, and Virginia Tech, "Preserving Symmetries for Variational Quantum Eigensolvers in the Presence of Noise," , 1-13 (2020), arXiv:arXiv:2003.00171v1. 
[34] Bryan T Gard, Linghua Zhu, George S Barron, Nicholas J Mayhall, Sophia E. Economou, and Edwin Barnes, "Efficient symmetry-preserving state preparation circuits for the variational quantum eigensolver algorithm," npj Quantum Inf. 6 (2020), 10.1038/s41534-019-02401, arXiv:1904.10910.

[35] Scott E. Smart and David A. Mazziotti, "Efficient two-electron ansatz for benchmarking quantum chemistry on a quantum computer," Phys. Rev. Res. 2, 023048 (2020).

[36] Jarrod R. McClean, Jonathan Romero, Ryan Babbush, and Alán Aspuru-Guzik, "The theory of variational hybrid quantum-classical algorithms," New Journal of Physics 18, 023023 (2016), arXiv:1509.04279.

[37] Abhinav Kandala, Antonio Mezzacapo, Kristan Temme, Maika Takita, Markus Brink, Jerry M. Chow, and Jay M. Gambetta, "Hardware-efficient variational quantum eigensolver for small molecules and quantum magnets," Nature 549, 242-246 (2017), arXiv:1704.05018.

[38] Ilya G. Ryabinkin, Scott N. Genin, and Artur F. Izmaylov, "Constrained Variational Quantum Eigensolver: Quantum Computer Search Engine in the Fock Space," Journal of Chemical Theory and Computation 15, 249-255 (2019), arXiv:1806.00461.

[39] Nikolaj Moll, Andreas Fuhrer, Peter Staar, and Ivano Tavernelli, "Optimizing qubit resources for quantum chemistry simulations in second quantization on a quantum computer," J. Phys. A: Math. Theor. 49 (2016), 10.1088/1751-8113/49/29/295301, arXiv:1510.04048.

[40] Sergey Bravyi, Jay M. Gambetta, Antonio Mezzacapo, and Kristan Temme, "Tapering off qubits to simulate fermionic Hamiltonians," , 1-15 (2017), arXiv:1701.08213.

[41] Kanav Setia, Richard Chen, Julia E. Rice, Antonio Mezzacapo, Marco Pistoia, and James Whitfield, "Reducing qubit requirements for quantum simulation using molecular point group symmetries," , 1-6 (2019), arXiv:1910.14644.

[42] Sam McArdle, Xiao Yuan, and Simon Benjamin, "Error-Mitigated Digital Quantum Simulation," Phys. Rev. Lett. 122, 180501 (2019), arXiv:1807.02467.

[43] X. Bonet-Monroig, R. Sagastizabal, M. Singh, and T. E. O'Brien, "Low-cost error mitigation by symmetry verification," Phys. Rev. A 98, 062339 (2018).

[44] R. Sagastizabal, X. Bonet-Monroig, M. Singh, M. A. Rol, C. C. Bultink, X. Fu, C. H. Price, V. P. Ostroukh, N. Muthusubramanian, A. Bruno, M. Beekman, N. Haider, T. E. O’Brien, and L. DiCarlo, "Experimental error mitigation via symmetry verification in a variational quantum eigensolver," Phys. Rev. A 100, 010302 (2019), arXiv:1902.11258. 
[45] Artur F. Izmaylov, Tzu-Ching Yen, and Ilya G. Ryabinkin, "Revising the measurement process in the variational quantum eigensolver: is it possible to reduce the number of separately measured operators?" Chem. Sci. 10, 3746-3755 (2019), arXiv:1810.11602.

[46] Artur F. Izmaylov, Tzu-Ching Yen, Robert A. Lang, and Vladyslav Verteletskyi, "Unitary Partitioning Approach to the Measurement Problem in the Variational Quantum Eigensolver Method," J. Chem. Theory Comput. 16, 190-195 (2020), arXiv:1907.09040.

[47] Pranav Gokhale, Olivia Angiuli, Yongshan Ding, Kaiwen Gui, Teague Tomesh, Martin Suchara, Margaret Martonosi, and Frederic T. Chong, "Minimizing State Preparations in Variational Quantum Eigensolver by Partitioning into Commuting Families," (2019), arXiv:1907.13623.

[48] P. Jordan and E. Wigner, "Über das Paulische Äquivalenzverbot," Z. Angew. Phys. 47, 631$651(1928)$.

[49] Andrew Tranter, Sarah Sofia, Jake Seeley, Michael Kaicher, Jarrod McClean, Ryan Babbush, Peter V. Coveney, Florian Mintert, Frank Wilhelm, and Peter J. Love, "The Bravyi-Kitaev transformation: Properties and applications," Int. J. Quantum Chem. 115, 1431-1441 (2015).

[50] Andrew Tranter, Peter J. Love, Florian Mintert, and Peter V. Coveney, "A Comparison of the Bravyi-Kitaev and Jordan-Wigner Transformations for the Quantum Simulation of Quantum Chemistry," J. Chem. Theory Comput. 14, 5617-5630 (2018), arXiv:1812.02233.

[51] Sergey B. Bravyi and Alexei Yu. Kitaev, "Fermionic Quantum Computation," Ann. Phys. 298, 210-226 (2002), arXiv:0003137 [quant-ph].

[52] Jarrod R. McClean, Mollie E. Schwartz, Jonathan Carter, and Wibe A. de Jong, "Hybrid Quantum-Classical Hierarchy for Mitigation of Decoherence and Determination of Excited States," Phys. Rev. A 95, 042308 (2016), arXiv:1603.05681.

[53] Pranav Gokhale and Frederic T. Chong, "O $\left(N^{3}\right)$ Measurement Cost for Variational Quantum Eigensolver on Molecular Hamiltonians," (2019), arXiv:1908.11857.

[54] Vladyslav Verteletskyi, Tzu-Ching Yen, and Artur F. Izmaylov, "Measurement Optimization in the Variational Quantum Eigensolver Using a Minimum Clique Cover," , 1-6 (2019), arXiv:1907.03358.

[55] Tiago P. Peixoto, "The graph-tool python library," figshare (2014), 10.6084/m9.figshare.1164194. 
[56] Héctor Abraham, AduOffei, Rochisha Agarwal, Ismail Yunus Akhalwaya, Gadi Aleksandrowicz, Thomas Alexander, Matthew Amy, Eli Arbel, Arijit02, Abraham Asfaw, Artur Avkhadiev, Carlos Azaustre, AzizNgoueya, Abhik Banerjee, Aman Bansal, Panagiotis Barkoutsos, George Barron, George S. Barron, Luciano Bello, and Yael Ben-Haim et al., "Qiskit: An open-source framework for quantum computing," (2019).

[57] Jens Koch, Terri M. Yu, Jay Gambetta, A. A. Houck, D. I. Schuster, J. Majer, Alexandre Blais, M. H. Devoret, S. M. Girvin, and R. J. Schoelkopf, "Charge-insensitive qubit design derived from the Cooper pair box," Phys. Rev. A 76, 042319 (2007).

[58] Jerry M. Chow, A. D. Córcoles, Jay M. Gambetta, Chad Rigetti, B. R. Johnson, John A. Smolin, J. R. Rozen, George A. Keefe, Mary B. Rothwell, Mark B. Ketchen, and M. Steffen, "Simple all-microwave entangling gate for fixed-frequency superconducting qubits," Phys. Rev. Lett. 107, 080502 (2011).

[59] Christopher J. Wood, Jacob D. Biamonte, and David G. Cory, "Tensor networks and graphical calculus for open quantum systems," Quantum Information and Computation 15, 759-811 (2015), arXiv:1111.6950.

[60] Cjwood, "How good is basic_device_noise_model() simulating the noise in the quantum computer?" (2019), https://quantumcomputing.stackexchange.com/questions/8958/how-goodis-basic-device-noise-model-simulating-the-noise-in-the-quantum-compu, accessed November 4th, 2020.

[61] Michael A. Nielsen and Isaac L. Chuang, Cambridge University Press (Cambridge University Press, Cambridge, 2010). 